\title{
Clinical Importance of Purulence in Methicillin- Resistant Staphylococcus aureus Skin and Soft Tissue Infections
}

\author{
Susan E. Crawford, MD, Michael Z. David, MD, PhD, Daniel Glikman, MD, \\ Kimberly J. King, MS, Susan Boyle-Vavra, PhD, and Robert S. Daum, MD
}

Background: The so-called community-associated methicillin-resistant Staphylococcus aureus (MRSA) strains are more frequently susceptible to non- $\beta$-lactam antibiotics (including clindamycin) than health care-associated MRSA strains. We assessed whether predictive clinical characteristics of presumptive MRSA infections can be identified to guide choice of empiric antibiotic therapy.

Methods: A clinical syndrome was assigned to each inpatient and outpatient at the University of Chicago Medical Center with an MRSA infection in 2004 to 2005. Antimicrobial susceptibilities and molecular characteristics of MRSA isolates were assessed. Patients were stratified by lesion characteristics.

Results: Of MRSA isolates from 262 patients with purulent skin and soft tissue infections (SSTIs), $231(88 \%)$ were susceptible to clindamycin, $253(97 \%)$ contained staphylococcal chromosomal cassette mec (SCCmec) IV, and 245 (94\%) contained Panton-Valentine leukocidin ( $p v l$ ) genes, characteristics associated with community-associated MRSA strains. The presence of a purulent SSTI had a positive predictive value of $88 \%$ for a clindamycin-susceptible MRSA isolate. Among 87 isolates from a nonpurulent SSTI, $44 \%$ were susceptible to clindamycin and $34 \%$ contained pvl genes. In 179 invasive MRSA disease isolates, $33 \%$ were clindamycin-susceptible and $26 \%$ carried $p v l$ genes.

Conclusions: A purulent MRSA SSTI strongly predicted the presence of a clindamycin-susceptible MRSA isolate. Presence of the $p v l$ genes was almost universal among MRSA isolates causing purulent SSTIs; this was less common in nonpurulent SSTIs and other clinical syndromes. (J Am Board Fam Med 2009;22:647-654.)

Methicillin-resistant Staphylococcus aureus (MRSA) isolates were first recognized in the $1960 \mathrm{~s}^{1,2}$ and

This article was externally peer reviewed.

Submitted 5 February 2009; revised 11 May 2009; accepted 19 May 2009.

From the Department of Pediatrics, The University of Chicago, IL (SEC, MZD, DG, KJK, SB-V, RSD); and the Infectious Disease Unit and Pediatrics, Western Galilee Hospital, Nahariya, Israel (DG).

Funding: Support has been provided by the Centers for Disease Control and Prevention (R01 CCR523379 and R01 C1000373-01), the National Institute of Allergy and Infectious Diseases (R01 A140481-01A1), and the Grant Healthcare Foundation (RSD, SB-V). Support has also been provided by Centers for Disease Control and Prevention grant R01 C1000373-01 (RSD, MZD) and National Institute of Allergy and Infectious Diseases grant 1R01A1067584-01A2 (SEC).

Conflict of interest: Grant funding has been provided to RSD from the National Institute of Allergy and Infectious Diseases, the Centers for Disease Control and Prevention, as well as Grant Healthcare Foundation, Sage Products, Inc., Pfizer, Sanofi Pasteur, BD, and Clorox. RSD has served on paid advisory boards for Clorox, GlaxoSmithKline, Pfizer, and the MRSA National Faculty Meeting (sponsored by Astellas and Theravance).

Corresponding author: Michael Z. David, $\mathrm{MD}, \mathrm{PhD}$, Section of Infectious Diseases, Department of Medicine, The University of Chicago, 5841 S. Maryland Avenue, MC 6054, Chicago, IL 60637 (E-mail: mdavid@medicine.bsd.uchicago.edu). were largely confined to patients with certain health care exposures. ${ }^{3}$ During the past 10 years, novel MRSA isolates have been recognized among previously healthy members of the community who often lack these health care-associated (HA) MRSA risk factors. ${ }^{4,5}$

Both clinical information and molecular isolate characteristics have been used to distinguish patients with HA MRSA and community-associated (CA) MRSA infections. ${ }^{6}$ HA MRSA isolates are typically resistant to multiple antimicrobial drugs, including clindamycin, and, in the United States, belong to multilocus sequence type (MLST) 5 and contain the methicillin resistance element staphylococcal chromosomal cassette mec (SCCmec) type II. ${ }^{7}$ In contrast, CA MRSA-type isolates are frequently susceptible to clindamycin (with some exceptions $)^{8,9}$; belong to MLST 8 or 1 ; contain SCCmec type IV or, rarely, type $\mathrm{V}$; and bear the genes for the Panton Valentine leukocidin $(p v l) .^{10-12}$

Skin and soft tissue infections (SSTIs) account for approximately $80 \%$ of infections caused by 
CA MRSA strains, ${ }^{13}$ although severe, life-threatening disease syndromes have also been described. ${ }^{5,14-16}$ HA MRSA-type isolates, in contrast, are seldom associated with purulent SSTIs but have instead been associated with catheterrelated bacteremia, postoperative wound infections, and infections with onset 48 hours or more after hospitalization. ${ }^{17}$

Recent analyses of MRSA infections with onset in the community have revealed a complex epidemiology with both HA and CA MRSA strains circulating in the community. ${ }^{18-20}$ Moreover, nosocomial MRSA infections and MRSA infections among patients with health care-related risk factors have been caused by various isolate types, including CA MRSA strains. ${ }^{21-25}$

The epidemiologic case definition of CA MRSA used by the Centers for Disease Control and Prevention (CDC) employs the lack of HA risk factors as follows: culture from an outpatient or within 48 hours of hospital admission and no history of hemodialysis, surgery, residence in a long-term care facility, or hospitalization during the previous 12 months; no indwelling catheter or percutaneous device at the time of culture; and no previous isolation of MRSA. We have shown, however, that application of this definition to a patient with an MRSA infection substantially underestimates the prevalence of infection caused by CA MRSA strains. ${ }^{20}$ Although the CDC criteria do accurately identify a portion of the CA MRSA isolate disease burden in the community, many patients with health care exposures (who would be classified as having HA MRSA) have disease caused by CA MRSA strains. This underestimate of disease caused by CA MRSA strains is important because the CA MRSA isolates are often susceptible to more classes of antimicrobial drugs than are HA MRSA strains.

As the complex epidemiology of MRSA disease has evolved, we hypothesized that there might be identifiable clinical features that would distinguish disease caused by CA MRSA strains from disease caused by HA MRSA strains and that would allow clinicians to choose appropriate initial empiric therapy for putative MRSA infections. In particular, we hypothesized that the presence of a purulent SSTI might be a useful predictor of a CA MRSA strain with clindamycin susceptibility in the era of epidemic MRSA disease.

\section{Methods}

\section{Setting}

Pediatric and adult patients served by the inpatient, outpatient and emergency department facilities at the University of Chicago Medical Center (UCMC) were asked to participate in this study, as described previously. ${ }^{20}$ The institution has 577 inpatient beds and 26,200 annual admissions. More than 71,000 visits are made to the emergency department annually. UCMC serves an inner city population and draws tertiary referrals from the surrounding region.

\section{Microbiologic Studies}

The UCMC Clinical Microbiology Laboratory prospectively identified all MRSA isolates collected between July 1, 2004, and June 30, 2005, from patients in all clinical settings, as previously reported. ${ }^{20}$ The antibiotic susceptibility profile of each isolate was determined by Vitek testing (bioMérieux Vitek, Hazelwood, MO) for oxacillin, clindamycin, erythromycin, and gentamicin. Isolates that were resistant to erythromycin but susceptible to clindamycin by Vitek testing underwent a $\mathrm{D}$ test to detect inducible clindamycin resistance. All assays were performed in accordance with Clinical and Laboratory Standards Institute guidelines. ${ }^{26}$

\section{Molecular Studies}

For all MRSA isolates, polymerase chain reaction assays for mecA, SCCmec type, $l u k F-P V$, and $l u k S-P V$ (which encode $p v l$ ) were conducted and MLST was determined, as described previously. ${ }^{20,27,28}$

\section{Patient Information}

A review of all consenting patients' electronic medical records-including hospital discharge summaries and radiographic, laboratory, pathology, operative, and outpatient clinic reports-was conducted and a clinical syndrome was assigned to each MRSA patient's isolate using all available data by 2 physician investigators (SEC and DG), as described previously. ${ }^{20}$ The clinical syndrome and molecular type were independently assigned without knowledge of the susceptibility or the molecular testing results on the isolate. This study was approved by the Institutional Review Board of the Biological Sciences Division of the University of Chicago. 


\section{Definitions}

In a previous report, ${ }^{20}$ we stratified isolates according to the site from which the culture was obtained, including skin and soft tissue, respiratory, blood, bone and joint, and the urinary tract. For this investigation, each patient's isolate was assigned a more detailed syndrome classification.

Among patients with isolates from SSTIs, a lesion requiring incision and drainage or a lesion with spontaneously draining purulent fluid (as described by the treating physician) was defined as an "abscess." This group included treating cliniciandesignated diagnoses of abscesses as well as carbuncles, furuncles, boils, and cellulitis with purulent drainage. The diagnosis of "pustule" was assigned when an isolate was obtained from a pus-containing, 1 to $2 \mathrm{~mm}$, superficial skin lesion. A chronic ulcer or an open pressure sore with nonpurulent drainage (if drainage was present) found in a patient with diabetes, peripheral vascular disease, or another underlying disease predisposing to these conditions was termed a "wound."

Patients with an isolate from the respiratory tract were assigned a diagnosis of ventilator-associated pneumonia, pneumonia with empyema, or other pneumonia. Any designation of MRSA pneumonia required the presence of respiratory symptoms such as cough, signs such as tachypnea and rales, consistent findings on a chest radiograph, and the prescription by the treating physician of an antibiotic effective against MRSA. Isolates colonizing the respiratory tract without attendant clinical illness and those obtained from the respiratory tract of patients with cystic fibrosis during routine surveillance were not included for analysis.

Some patients had several foci of infection. An identified anatomic focus of infection was assigned priority in designating the syndrome. For example, a patient with bacteremia and osteomyelitis was categorized as having osteomyelitis. Bacteremia without a focus of infection was termed "bacteremia without focus."

An isolate that was shown to be susceptible to clindamycin by Vitek testing (bioMérieux Vitek), resistant to erythromycin, and showed a positive D test was considered resistant to clindamycin.

\section{Statistical Analysis}

Categorical data were compared using the $\chi^{2}$ or Fisher exact tests, as appropriate. All calculations were performed using Stata 9.0 (StataCorp, Col- lege Station, TX). $P<.05$ was considered significant.

\section{Results}

The UCMC Clinical Microbiology Laboratories identified 1225 MRSA isolates during the study period. After excluding multiple isolates from the same patient, confirming the species as $S$. aureus and the presence of $\operatorname{mec} A$, as well as excluding respiratory colonization or skin surveillance culture isolates and the 35 patients who declined to participate, 548 MRSA isolates were designated for further analysis.

Of these 548 MRSA isolates, 362 (66\%) contained SCCmec type IV and 321 (89\%) of these isolates were pol positive. Three hundred sixteen (87\%) of the isolates containing SCCmec type IV were susceptible to clindamycin. Among the 46 SCCmec type IV isolates resistant to clindamycin, $21(46 \%)$ were detected by single-agent testing and 25 (54\%) were detected by D test. Among SCCmec IV-bearing isolates, 319 (88\%) were ST8, 20 (5\%) were ST1, and $11(3 \%)$ were ST5.

One hundred seventy-nine (33\%) contained SCCmec type II. None of the 179 were pol positive and $9(5 \%)$ were susceptible to clindamycin. Among the isolates resistant to clindamycin, 152 $(85 \%)$ were resistant on single-agent testing and 18 (10\%) showed a positive D test. By MLST, 154 (86\%) SCCmec II-bearing isolates were ST5, 14 (8\%) were ST231, and only 3 (2\%) were ST8.

Seven isolates had a SCCmec element that could not be classified as SCCmec types I through VI. None of them were pvl positive; all belonged to ST5 and were isolated from patients with one or more HA risk factors. One was susceptible to clindamycin.

A site of isolation and specific syndrome was identified for each of the 548 MRSA isolates, as seen in Tables 1 and 2. The designation of "abscess" strongly predicted the presence of a clindamycin-susceptible, SCCmec type IV-containing, pvl positive isolate (Table 2). Two hundred eight (89\%) of the 234 MRSA abscess isolates were susceptible to clindamycin; SCCmec type IV was found in 229 of these $(98 \%)$ and $p v l$ genes were found in 223 (95\%). Of the 229 SCCmec type IV-containing isolates found in abscesses, only 6 (2.6\%) lacked the pol genes.

Twenty-eight MRSA isolates were obtained from patients with a purulent SSTI that was not 
Table 1. Characteristics of Methicillin-Resistant Staphylococcus aureus Isolates from Patients Without Skin and Soft Tissue Infections $(\mathrm{n}=199)$

\begin{tabular}{|c|c|c|c|c|}
\hline Syndrome & $\begin{array}{l}\text { Strains Tested } \\
\quad(\mathrm{n}=199)\end{array}$ & $\begin{array}{l}\text { Strains Susceptible to } \\
\text { Clindamycin }\end{array}$ & $\begin{array}{l}\text { Strains Containing } \\
\text { SCCmec IV }\end{array}$ & $\begin{array}{l}\text { Strains with the } \\
P V L \text { Genes }\end{array}$ \\
\hline Respiratory tract* & $63(32)$ & $13(21)$ & $16(25)$ & $13(21)$ \\
\hline Pneumonia & 26 & $5(19)$ & $6(23)$ & $5(19)$ \\
\hline Empyema & 4 & $2(50)$ & $2(50)$ & $2(50)$ \\
\hline Ventilator-associated pneumonia & 19 & $4(21)$ & $5(26)$ & $4(21)$ \\
\hline Other respiratory isolates $^{\dagger}$ & $14^{\ddagger}$ & $2(14)$ & $3(21)$ & $2(14)$ \\
\hline Bacteremia syndromes & $60(30)$ & $14(23)$ & $19(32)$ & $11(18)$ \\
\hline Bacteremia & 18 & $4(22)$ & $6(33)$ & $5(28)$ \\
\hline Line infection & $38^{\ddagger}$ & $8(21)$ & $10(26)$ & $4(11)$ \\
\hline Endocarditis $^{\S}$ & 4 & $2(50)$ & $2(50)$ & $2(50)$ \\
\hline Bone and joint & $33(17)$ & $16(48)$ & $17(52)$ & $13(39)$ \\
\hline Osteomyelitis & $18^{\ddagger}$ & $7(39)$ & $9(50)$ & $7(39)$ \\
\hline Septic arthritis & 12 & $7(58)$ & $7(58)$ & $5(42)$ \\
\hline Septic bursitis & 3 & $2(66)$ & $1(33)$ & $1(33)$ \\
\hline Urinary tract & $23(12)$ & $5(22)$ & $4(17)$ & $3(13)$ \\
\hline Other" & $20(10)$ & $9(45)$ & $9(45)$ & $5(25)$ \\
\hline
\end{tabular}

All data provided as $\mathrm{n}(\%)$.

*Isolates from the upper respiratory tract designated as colonization isolates were excluded; isolates from patients with cystic fibrosis were also excluded.

${ }^{\dagger}$ Includes isolates obtained from patients with bronchitis, chronic laryngitis, sinusitis, tracheitis, and acute otitis media.

${ }^{\ddagger}$ Isolates with an untypeable SCCmec element were included.

${ }^{\S}$ As defined by modified Duke criteria.

"Including intra-abdominal fluid, deep intraperitoneal abscess, gall bladder, pericardial fluid, conjunctiva or the external ear canal. PVL, Panton-Valentine leukocidin.

designated as an abscess. The syndromes for these patients were designated as a pustule, paronychia, pyomyositis, or felon. Twenty-three ( $82 \%)$ of these nonabscess purulent MRSA SSTI isolates were susceptible to clindamycin; 24 (86\%) contained SCCmec type IV and $22(79 \%)$ were pol positive.

Table 2. Characteristics of Methicillin-Resistant Staphylococcus aureus Isolates from Patients with Skin and Soft Tissue Infections $(\mathrm{n}=349)$

\begin{tabular}{lcccc}
\hline Type of Infection & $\begin{array}{c}\text { Strains Tested } \\
(\mathrm{n}=349)\end{array}$ & $\begin{array}{c}\text { Strains Susceptible to } \\
\text { Clindamycin }\end{array}$ & $\begin{array}{c}\text { Strains Containing } \\
\text { SCCmec IV }\end{array}$ & $\begin{array}{c}\text { Strains with the } \\
P V L \text { Genes }\end{array}$ \\
\hline Purulent SSTI & $262(75)$ & $231(88)$ & $253(97)$ & $245(94)$ \\
$\quad$ Abscess & 234 & $208(89)$ & $229(98)$ & $223(95)$ \\
Pustules & 16 & $14(88)$ & $13(81)$ & $13(81)$ \\
Paronychia & 4 & $3(75)$ & $4(100)$ & $3(75)$ \\
Pyomyositis & 6 & $4(67)$ & $5(83)$ & $4(67)$ \\
Felon & 2 & $2(100)$ & $2(100)$ & $2(100)$ \\
Nonpurulent SSTI & $87(25)$ & $38(44)$ & $14(51)$ & $30(34)$ \\
Postoperative wound infection & $42^{*}$ & $15(36)$ & $9(38)$ & $13(24)$ \\
Wound & 17 & $8(47)$ & $12(67)$ & $5(29)$ \\
Wound and foreign body & $18^{*}$ & $2(25)$ & $1(63)$ & $9(50)$ \\
Burns & 8 & $1(50)$ & $1(50)$ & $5(63)$ \\
Impetigo & 2 & & $1(50)$ \\
\hline
\end{tabular}

All data provided as $\mathrm{n}(\%)$.

${ }^{*}$ Isolates with an untypeable SCCmec element were included.

SSTI, skin and soft tissue infenction; PVL, Panton-Valentine leukocidin. 
Table 3. Characteristics of Methicillin-resistant Staphylococcus aureus Isolates from Patients with Purulent and Nonpurulent Skin and Soft Tissue Infections

\begin{tabular}{lccr}
\hline Characteristic & $\begin{array}{c}\text { Purulent SSTI* } \\
(\mathrm{n}=262)\end{array}$ & $\begin{array}{c}\text { Nonpurulent SSTI* } \\
(\mathrm{n}=87)\end{array}$ & $P$ \\
\hline Contains SCCmec IV & $253(97)$ & $44(51)$ & $<.001$ \\
Contains SCCmec II & $9(3)$ & $41(47)$ & $<.001$ \\
Positive for PVL genes & $245(94)$ & $30(34)$ & $<.001$ \\
Susceptibility to: & & & $<.001$ \\
Clindamycin & $231(88)$ & $38(44)$ & $<.001$ \\
Ciprofloxacin & $237(90)$ & $31(36)$ & .001 \\
Gentamicin & $261(99)$ & $77(89)$ & .1 \\
Erythromycin & $19(7)$ & $11(13)$ & .04 \\
Rifampin & $260(99)$ & $83(95)$ & .1 \\
Trimethoprim-sulfamethoxazole & $232(100)$ & $31(97)$ & \\
\hline
\end{tabular}

*Data provided as n (\%).

${ }^{\dagger}$ Two hundred thirty-two isolates from purulent SSTIs and 32 from nonpurulent SSTIs were tested for susceptibility to trimethoprim-sulfamethoxazole.

SSTI, skin and soft tissue infenction; PVL, Panton-Valentine leukocidin.

When clinical data regarding abscess and nonabscess purulent SSTIs were pooled, 88\% were caused by MRSA isolates that were susceptible to clindamycin, 97\% contained SCCmec IV, and 94\% were pol positive. Eighty-nine percent of purulent SSTI isolates belonged to ST8, 5\% belonged to ST1, and $4 \%$ belonged to ST5; $2 \%$ of isolates belonged to other MLST types. Overall, the positive predictive value of a purulent SSTI for clindamycin susceptibility was $88 \%$. The high percent of clindamycin-susceptible strains in all purulent SSTI isolates did not differ significantly $(P=.37)$ among isolates obtained from children or adults.

The 87 MRSA isolates from nonpurulent SSTIs were obtained from patients with infected wounds in the presence or absence of an associated foreign body (eg, nonpurulent drainage at a central venous catheter insertion site), a burn, impetigo, or a postoperative wound infection. The isolates from these patients contained SCCmec types II and IV with approximately equal frequency ( $47 \%$ and $51 \%$, respectively); 38 (44\%) were susceptible to clindamycin and 30 (34\%) were pvl positive. ST5 was represented among $47 \%$ of nonpurulent SSTI isolates; ST8 was found in $41 \%$ of the isolates and $12 \%$ of isolates had other MLST types. Clindamycin susceptibility was not reliably predicted by clinical syndrome among patients with a nonpurulent SSTI (Table 3).

It is noteworthy that $97 \%$ of the SCCmec IVcontaining isolates found in patients with purulent SSTIs contained the pol genes compared with only
30 of $44(68 \%)$ of the isolates that contained SCCmec IV obtained from patients with nonpurulent SSTIs $(P<.001)$.

Among isolates associated with disease from the respiratory tract, bloodstream, bone or joint, or the urinary tract, we found that none of these nonSSTI syndromes were strongly associated with clindamycin susceptibility, pvl gene carriage, or SCCmec type IV (Table 1).

A patient with isolation of MRSA from a normally sterile body site-including blood, pleural fluid, joint/synovial fluid, or bone-was considered to have invasive MRSA disease. ${ }^{11}$ Of the 95 isolates from patients with invasive MRSA disease, 31 (33\%) were susceptible to clindamycin, 37 (39\%) contained SCCmec type IV, and 25 (26\%) carried the pol genes.

Using the CDC case definition for CA MRSA, only 228 of 390 (58\%) patients with pvl-positive isolates that contained SCCmec type IV would have been classified as CA MRSA, whereas 162 (42\%) of the patients with $p v l$-positive isolates that contained SCCmec type IV would have been classified as HA MRSA cases.

Of the patients with a purulent MRSA SSTI $(\mathrm{n}=262), 91(35 \%)$ had a health care risk factor that would result in their isolate being classified as HA MRSA by the CDC definition. Of these 91 isolates, $74(81 \%)$ were susceptible to clindamycin, $82(90 \%)$ possessed the pvl genes, and 85 (93\%) contained SCCmec type IV. 
Table 4. Comparison of Purulent and Nonpurulent Skin and Soft Tissue Infections among Patients with One or More Health Care Risk Factors as Defined by the Centers for Disease Control and Prevention $(n=169)$

\begin{tabular}{lccr}
\hline Characteristic & Purulent SSTI* $(\mathrm{n}=91)$ & Nonpurulent SSTI* $(\mathrm{n}=78)$ & $P$ \\
\hline Contains SCCmec IV & $85(93)$ & $36(46)$ & $<.001$ \\
Contains SCCmec II & $6(7)$ & $40(51)$ & $<.001$ \\
Positive for PVL genes & $82(90)$ & $23(29)$ & $<.001$ \\
Susceptible to clindamycin & $74(81)$ & $32(41)$ & $<.001$ \\
\hline
\end{tabular}

*All data provided as $\mathrm{n}(\%)$.

SSTI, skin and soft tissue infenction; $P V L$, Panton-Valentine leukocidin.

In contrast, among the 87 isolates from patients with a nonpurulent SSTI, 78 (90\%) were identified as HA MRSA by CDC criteria. Of these, 32 (41\%) were susceptible to clindamycin, 36 (46\%) contained SCCmec type IV, and 23 (29\%) carried the pol genes. Thus, even among those patients with a CDC-defined health care risk factor, those with a purulent MRSA SSTI were significantly more likely to have a clindamycin-susceptible, pvl-positive, SCCmec type IV isolate than patients with nonpurulent SSTIs $(P<.001$; Table 4).

\section{Discussion}

The presence of a purulent SSTI strongly predicted the presence of an MRSA isolate that was susceptible to clindamycin, contained SCCmec type $\mathrm{IV}$, and possessed the pol genes, regardless of the presence of CDC health care risk factors. In contrast, among the patients with nonpurulent SSTIs with respiratory, bone and joint, bloodstream, and urinary tract infections from which MRSA was isolated, strains containing both SCCmec types II and IV were responsible for infection.

The absence of a CDC health care risk factor also identified a subset of patients whose MRSA isolate was susceptible to clindamycin and contained SCCmec IV and the pol genes. ${ }^{20}$ However, the CDC criteria for CA MRSA identified a smaller subset of patients for whom clindamycin therapy would be effective.

Thirteen percent of strains that contained SCCmec type IV and 11\% of MRSA isolated from purulent SSTIs were resistant to clindamycin. This rate of clindamycin resistance among CA MRSA strains is comparable with that reported by others, ${ }^{29}$ but the rate varies geographically and temporally. ${ }^{30}$ Clindamycin has been suggested as firstline therapy in the treatment of putative staphylococcal SSTIs in areas in which MRSA strains comprise $>10 \%$ of $S$. aureus infections. ${ }^{31}$ Clindamycin is often effective in the treatment of infections caused by MRSA strains possessing the inducible form of clindamycin resistance. However, a few failures have been reported, ${ }^{32}$ and for this reason we considered isolates with a positive $\mathrm{D}$ test as resistant to clindamycin. Alternatives for the empiric treatment of CA MRSA SSTIs must be considered should the rate of clindamycin resistance increase; some consider trimethoprim-sulfamethoxazole (TMP/SMX) as first-line therapy for this reason and because of its better tolerability in some cases, ${ }^{33}$ although few reported data attest to its efficacy. Indeed, we found TMP/SMX susceptibility to be near universal in isolates tested from both purulent and nonpurulent SSTIs. Therapy with TMP/SMX is not recommended when infection caused by group A Streptococcus is likely.

Caution must be used when trying to interpret institution-specific rates of clindamycin resistance among MRSA isolates. Antibiograms often reflect susceptibility among pooled MRSA isolates and do not distinguish between isolates that contain SCCmec IV that are usually susceptible to clindamycin and those isolates that contain SCCmec II that are usually resistant to clindamycin. Institutions may find it helpful to stratify reporting of MRSA antibiograms by clinical syndromes. Such stratification would be helpful for practitioners when monitoring antibiotic susceptibility patterns and serve as a practical guide in the treatment options for MRSA infections.

It has been suggested that pol might be an important virulence factor in CA MRSA disease. However, animal infection studies have provided contradictory results. ${ }^{34,35}$ Our data show near universality of $p v l$ genes in isolates from purulent SSTIs and their substantially lower prevalence in all other MRSA clinical syndromes. Of note, we 
also found that pol genes were significantly more common among strains that contain SCCmec type IV found in purulent SSTIs than in those strains containing SCCmec IV found in nonpurulent SSTIs or non-SSTI syndromes. This suggests that pol may not solely be a marker for SCCmec type IV strains but that it also may be important in the pathogenesis of purulent lesions characteristic of certain CA MRSA infections. ${ }^{35,36}$ Further studies are needed to delineate the role of $p v l$ in the pathogenesis of MRSA infections.

Our study has several limitations. We only studied infections caused by MRSA and did not include those caused by methicillin-sensitive $S$. aureus. Our study represents clinical and isolate data from one center and may not be representative of other areas. The burden of community and hospital disease caused by MRSA was high in our center (approximately $60 \%$ of $S$. aureus isolates), but it is also high in many other US medical centers. ${ }^{30}$ Antibiotic resistance rates among CA MRSA isolates may have changed since this study period. In addition, clinical features of methicillin-sensitive $S$. aureus and MRSA infections have been found to be indistinguishable. ${ }^{37}$ Therefore, a putative $S$. aureus infection can be presumed to be MRSA and treated as such until culture results are obtained, but this approach may vary geographically.

We relied on physician documentation to assign a clinical syndrome category and assigned these syndromes retrospectively. For example, furuncles, carbuncles, and superficial primary cutaneous abscesses were grouped together as "abscess" because the detail provided by observer description made it difficult to stratify them further. Drainage from a postoperative wound infection or around a central venous catheter or another foreign body was often not described in sufficient detail. Therefore, if purulence was not described, we assigned the designation "nonpurulent." It is not known if such drainage, if purulent, would also predict the presence of an isolate that was susceptible to clindamycin, that contained SCCmec IV, and that was pol positive.

\section{Conclusion}

The diagnosis of a purulent, putative MRSA skin lesion predicts the presence of an MRSA isolate susceptible to clindamycin, bearing the SCCmec type IV element, and carrying pol genes, regardless of the presence of HA risk factors. Epidemiologic studies of MRSA infections that excluded patients with health care risk factors have probably underestimated the burden of clindamycin-susceptible MRSA disease. ${ }^{13}$ Recognition of a purulent SSTI will permit clinicians to select from a broader range of empiric antimicrobial drugs including clindamycin (when such therapy is deemed necessary), even among patients with CDC-defined, health carerelated risk factors.

\section{References}

1. Barber M. Methicillin-resistant staphylococci. J Clin Pathol 1961;14:385-93.

2. Barrett FF, McGehee RF, Finland M. Methicillinresistant Staphylococcus aureus at Boston City Hospital. N Eng J Med 1968;279:441-8.

3. Doebbeling BN. The epidemiology of methicillinresistant Staphylococcus aureus colonisation and infection. J Chemother 1995;7(Suppl 3):99-103.

4. Herold BC, Immergluck LC, Maranan MC, et al. Community-acquired methicillin-resistant Staphylococcus aureus in children with no identified predisposing risk. JAMA 1998;279:593-8.

5. Centers for Disease Control and Prevention. Four pediatric deaths from community-acquired methicillin-resistant Staphylococcus aureus-Minnesota and North Dakota, 1997-1999. MMWR Morb Mortal Wkly Rep 1999;48:707-10.

6. Naimi TS, LeDell KH, Como-Sabetti K, et al. Comparison of community- and health care-associated methicillin-resistant Staphylococcus aureus infection. JAMA 2003;290:2976-84.

7. Ito T, Katayama Y, Asada K, et al. Structural comparison of three types of staphylococcal cassette chromosome mec integrated in the chromosome in methicillin-resistant Staphylococcus aureus. Antimicrob Agents Chemother 2001;45:1323-36.

8. Diep BA, Chambers HF, Graber CJ, et al. Emergence of multidrug-resistant, community-associated, methicillin-resistant Staphylococcus aureus clone USA300 in men who have sex with men. Ann Intern Med 2008; 148:249-57.

9. Han LL, McDougal LK, Gorwitz RJ, et al. High frequencies of clindamycin and tetracycline resistance in methicillin-resistant Staphylococcus aureus pulsed-field type USA300 isolates collected at a Boston ambulatory health center. J Clin Microbiol 2007; 45:1350-2.

10. Ma XX, Ito T, Tiensasitorn C, et al. Novel type of staphylococcal cassette chromosome mec identified in community-acquired methicillin-resistant Staphylococcus aureus strains. Antimicrob Agents Chemother 2002;46:1147-52.

11. Daum RS, Ito T, Hiramatsu K, et al. A novel methicillin-resistance cassette in community-acquired me- 
thicillin-resistant Staphylococcus aureus isolates of diverse genetic backgrounds. J Infect Dis 2002;186:1344-7.

12. Vandenesch F, Naimi T, Enright MC, et al. Community-acquired methicillin-resistant Staphylococcus aureus carrying Panton-Valentine leukocidin genes: worldwide emergence. Emerg Infect Dis 2003;9:978-84.

13. Fridkin SK, Hageman JC, Morrison M, et al. Active Bacterial Core Surveillance Program of the Emerging Infections Program Network. Methicillin-resistant Staphylococcus aureus disease in three communities. N Engl J Med 2005;352:1436-44.

14. Adem PV, Montgomery CP, Husain AN, et al. Staphylococcus aureus sepsis and the WaterhouseFriderichsen syndrome in children. N Engl J Med 2005;353:1245-51.

15. Francis JS, Doherty MC, Lopatin U, et al. Severe community-onset pneumonia in healthy adults caused by methicillin-resistant Staphylococcus aureus carrying the Panton-Valentine leukocidin genes. Clin Infect Dis 2005;40:100-7.

16. Miller LG, Perdreau-Remington F, Rieg G, et al. Necrotizing fasciitis caused by community-associated methicillin-resistant Staphylococcus aureus in Los Angeles. N Engl J Med 2005;352:1445-53.

17. Diekema DJ, Pfaller MA, Schmitz FJ, et al. Survey of infections due to Staphylococcus species: frequency of occurrence and antimicrobial susceptibility of isolates collected in the United States, Canada, Latin America, Europe, and the Western Pacific region for the SENTRY Antimicrobial Surveillance Program, 1997-1999. Clin Infect Dis 2001;32(Suppl):S114-32.

18. Charlebois ED, Perdreau-Remington F, Kreiswirth $\mathrm{B}$, et al. Origins of community strains of methicillinresistant Staphylococcus aureus. Clin Infect Dis 2004; 39:47-54.

19. Klevens RM, Morrison MA, Nadle J, et al. Active Bacterial Core Surveillance MRSA Investigators. Invasive methicillin-resistant Staphylococcus aureus infections in the United States. JAMA 2007;298:1763-71.

20. David MZ, Glikman D, Crawford SE, et al. What is community-associated methicillin-resistant Staphylococcus aureus? J Infect Dis 2008;197:1235-43.

21. Maree CL, Daum RS, Boyle-Vavra S, Matayoshi K, Miller LG. Community-associated methicillin-resistant Staphylococcus aureus isolates causing healthcare-associated infections. Emerg Infect Dis 2007; 13:236-42.

22. Gonzalez BE, Rueda AM, Shelburne SA 3rd, Musher DM, Hamill RJ, Hulten KG. Community-associated strains of methicillin-resistant Staphylococcus aureus as the cause of healthcare-associated infection. Infect Control Hosp Epidemiol 2006;27:1051-6.

23. Healy CM, Hulten KG, Palazzi DL, Campbell JR, Baker CJ. Emergence of new strains of methicillinresistant Stapbylococcus aureus in a neonatal intensive care unit. Clin Infect Dis 2004;39:1460-6.

24. Saiman L, O'Keefe M, Graham PL 3rd, et al. Hospital transmission of community-acquired methicil- lin-resistant Staphylococcus aureus among postpartum women. Clin Infect Dis 2003;37:1313-9.

25. Klevens RM, Morrison MA, Fridkin SK, et al. Community-associated methicillin-resistant Staphylococcus aureus and healthcare risk factors. Emerg Infect Dis 2006;12:1991-3.

26. National Committee for Clinical Laboratory Standards. Performance standards for antimicrobial disk susceptibility testing. 14th Informational Supplement. Villanova, PA: National Committee for Clinical Laboratory Standards; 2004.

27. David MZ, Crawford SE, Boyle-Vavra S, Hostetler MA, Kim DC, Daum RS. Contrasting pediatric and adult methicillin-resistant Staphylococcus aureus isolates. Emerg Infect Dis 2006;12:631-7.

28. Boyle-Vavra S, Ereshefsky B, Wang CC, Daum RS. Successful multiresistant community-associated methicillin-resistant Staphylococcus aureus lineage from Taipei, Taiwan, that carries either the novel Staphylcoccal chromosome cassette (SCCmec) type VT or SCCmec type IV. J Clin Micro 2005;43:4719-30.

29. Kaplan SL, Hulten KG, Gonzalez BE, et al. Threeyear surveillance of community-acquired Staphylococcus aureus infections in children. Clin Infect Dis 2005;40:1785-91.

30. Moran GJ, Krishnadasan A, Gorwitz RJ, et al. Methicillin-resistant $S$. aureus infections among patients in the emergency department. N Engl J Med 2006; 355:666-74.

31. Daum RS. Skin and soft-tissue infections caused by methicillin-resistant Staphylococcus aureus. N Engl J Med 2007;357:380-90.

32. Frank AL, Marcinak JF, Mangat PD, et al. Clindamycin treatment of methicillin-resistant Stapbylococcus aureus infections in children. Pediatr Infect Dis J 2002;21:530-4.

33. Szumowski JD, Cohen DE, Kanaya F, Mayer KH. Treatment and outcomes of infections by methicillin-resistant Staphylococcus aureus at an ambulatory clinic. Antimicrob Agents Chemother 2007;51:423-8.

34. Voyich JM, Otto M, Mathema B, et al. Is PantonValentine leukocidin the major virulence determinant in community-associated methicillin-resistant Staphylococcus aureus disease? J Infect Dis 2006;194: 1761-70.

35. Labandeira-Rey M, Couzon F, Boisset S, et al. Staphylococcus aureus Panton-Valentine leukocidin causes necrotizing pneumonia. Science 2007;23:1130-3.

36. Ward PD, Turner WH. Identification of staphylococcal Panton-Valentine leukocidin as a potent dermonecrotic toxin. Infect Immun 1980;28:393-7.

37. Miller LG, Perdreau-Remington F, Bayer AS, et al. Clinical and epidemiologic characteristics cannot distinguish community-associated methicillin-resistant Staphylococcus aureus infection from methicillinsusceptible $S$. aureus infection: a prospective investigation. Clin Infect Dis 2007;44:471-82. 